\title{
Pachymeningeal en Plaque Tuberculoma in Children: A Case Report
}

\author{
Ibrahima Berete ${ }^{1,2 *}$, Alpha Boubacar Bah ${ }^{2}$, Seylan Diawara², \\ Mohammed Benzagmout ${ }^{1}$, Khalid Chakour ${ }^{1}$, \\ Mohammed Faiz Chaoui ${ }^{1}$
}

\author{
${ }^{1}$ Service de Neurochirurgie CHU Hassan II, Fès, Morocco \\ ${ }^{2}$ Service de Neurochirurgie CHU Donka, Conakry, Guinea \\ Email:^iberete902@icloud.com
}

How to cite this paper: Berete, I., Bah, A.B., Diawara, S., Benzagmout, M., Chakour, K. and Chaoui, M.F. (2020) Pachymeningeal en Plaque Tuberculoma in Children: A Case Report. Open Journal of Modern Neurosurgery, 10, 63-69.

https://doi.org/10.4236/ojmn.2020.101007

Received: November 1, 2019

Accepted: December 2, 2019

Published: December 5, 2019

Copyright $\odot 2020$ by author(s) and Scientific Research Publishing Inc. This work is licensed under the Creative Commons Attribution International License (CC BY 4.0).

http://creativecommons.org/licenses/by/4.0/

\begin{abstract}
Pachymeningeal en plaque tuberculoma is a rare manifestation of tuberculosis and has a poor prognosis if not diagnosed early and treated adequately. The aim of the present paper is to collect the reported clinical and radiological signs in the literature and compare it with a personal observation to evaluate the possibility of a presumptive diagnosis. A 4-year-old boy presented at the pediatric emergency department with 7 days history of severe headache, vomiting blurred vision and had one partial seizure. His neurologic examination was notable for decreased visual acuity with bilateral papilledema and intact extra-ocular muscles. His neck was rigid. Neuroimaging revealed a multifocal dural-based mass on the fronto-parietal convexity with dense heterogeneous contrast enhancement and hydrocephalus. He has undergone a Ventriculoperitoneal shunt and surgical excision of the mass the next day. Outcome was favorable with antituberculosis therapy and corticosteroids. The clinical presentation and outcome were similar to the reported cases in the literature.
\end{abstract}

\section{Keywords}

Tuberculoma, Pachymeningeal, Intracranial, Tuberculosis, MRI, CT Scan

\section{Introduction}

Pachymeningeal tuberculoma is a rare localization of neuro-meningeal tuberculosis (TB) secondary to pachymeningitis due to an infection with mycobacterium tuberculosis [1]. Pachymeningitis has been described as diffuse or localized thickening of the cranial dura mater with or without associated inflammation 
[2]. The damage to the central nervous system is multiple and variable. Progressive nature of the disease can lead to chronic headache with focal neurological signs due to compression from the thickened dura [3]. Clinical manifestations vary depending on the location of the pathology, and the affected patients commonly present with manifestations of increased intracranial pressure, such as headache, or progressive neurologic deficits arising due to compression of neural structures by the thickened dura mater such as cranial neuropathies including vestibular involvement and seizures [2]. TB meningitis still remains the most common manifestation, affecting mainly children and adolescents [3]. Their radiological identification has been improved by the evolution of imaging techniques, but their diagnosis is suspected in the majority of cases during surgery secondarily confirmed by pathological study [4]. The aim of the present work was to gather clinical and neuroradiological arguments that could evoke the diagnosis of this particular localization of neuro-meningeal tuberculosis through a review of similar cases reported to date in the literature.

\section{Observation}

A 4-year-old patient presented in our emergency room with complaints of headaches, partial tonico-clonic seizure episodes, vomiting, intermittent fever and blurred vision for 7 days. His vaccination was up-to date and he had no history of trauma, chronic cough and no past history of any major illness. His family history was unremarkable.

On examination, the patient was febrile (38.5-degree Celcius), conscious and oriented to person, place and time. He was hypotonic and his conjunctives were pale. He had full strength on all extremities with a decreased tone, bilateral papilledema, decreased visual acuity and neck stiffness.

Routine investigations showed hypochromic anemia and a normal chest $\mathrm{X}$ ray. A Brain Computed Tomography scan was done and showed an irregular multifocal ill-defined extra axial isodense lesion over right fronto-parieto-temporal convexity with heterogenous contrast enhancement, a perilesional edema, calcification and triventricular hydrocephalus (Figure 1). On the Magnetic resonance Imaging (MRI) the lesion was multifocal hypointense on T2 weighted, iso-intense on T1 weighted with heterogeneous enhancement on gadolinium contrast and hydrocephalus (Figure 2).

A presumptive diagnosis of tuberculoma was made and a surgery for ventriculo-peritoneal shunting was performed with a normal Cerebro-Spinal Fluid (CSF) bacteriological study. A second procedure was planned for removal of the tumor. During surgery we found an extra-axial en plaque mass (Comment Number 3 clarification: Extra-axial tumors are lesions, neoplastic and not, which are external to the brain parenchyma and can originate in the skull, meninges, cranial nerves, and brain appendages such as the pituitary gland. An En plaque mass is a diffuse mass with broad base on the dura) which was firm, slightly yellowish and fibrous, adherent to the internal layer of the dura and the parietal 

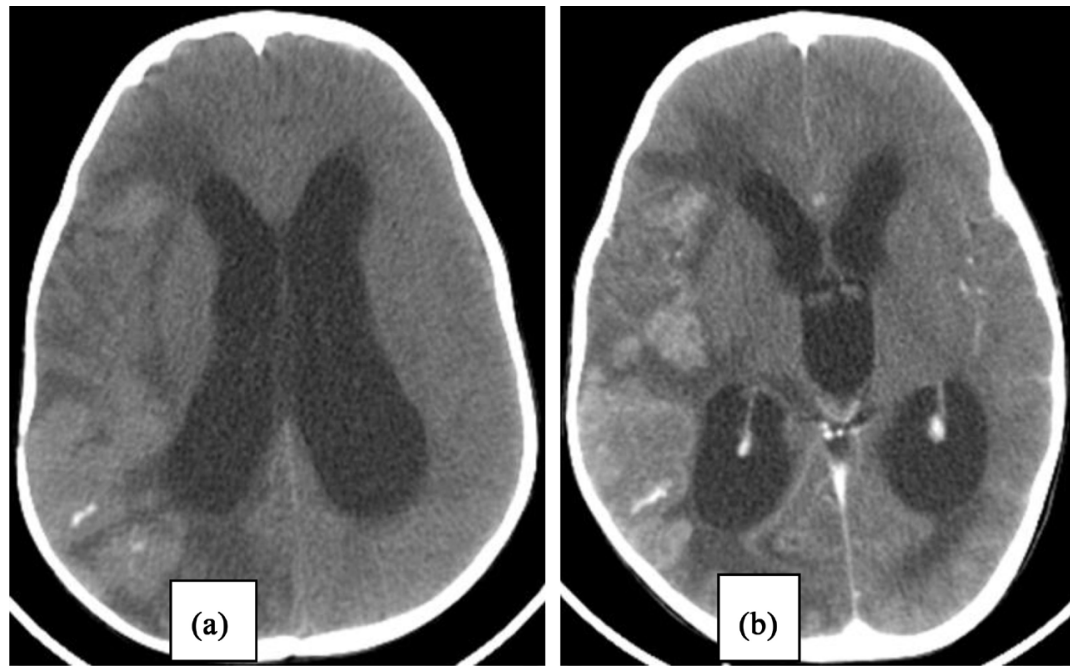

Figure 1. CT scan without (a) and with contrast (b) demonstrated a right parieto-temporal multifocal mass lesion with a calcification and heterogenous enhancement with contrast and hydrocephalus.

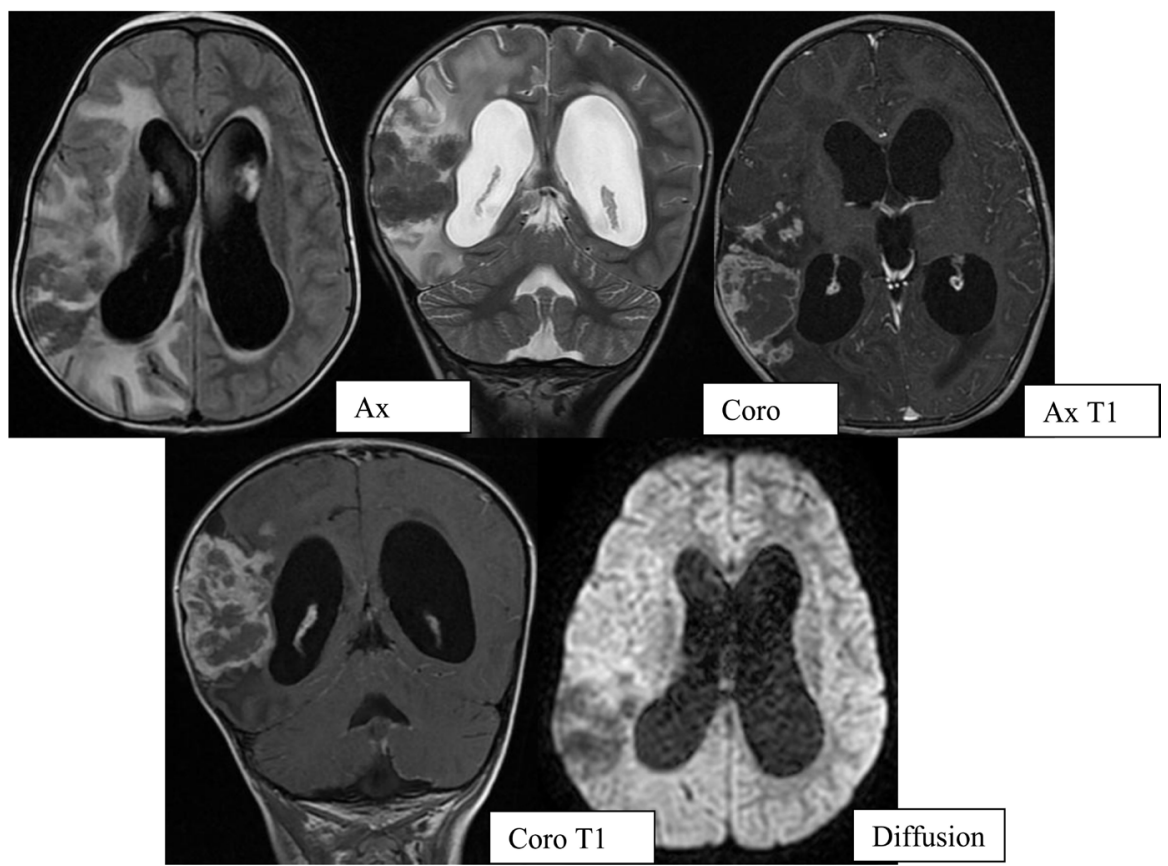

Figure 2. Brain MRI, the lesion is hypointense on T1 weighted and diffusion with heterogeneous contrast enhancement with gadolinium and perilesional hyperintensity on T2 weighted consistent with edema.

convexity with a thick capsule. The lesion was excised with part of the adherent dura. The overlying bone was normal. The histopathological examination showed multiple necrotic areas, giant and epithelioid cells (Figure 3) and the Ziehl Neelsen staining showed the presence of acid-fast bacilli confirming the diagnosis of tuberculoma. The post-operative CT scan showed a subtotal resection of the en Plaque parietal mass. Postoperative recovery was uneventful. The patient was started on anti-tuberculosis therapy, Sodium Valproate and corticosteroids 


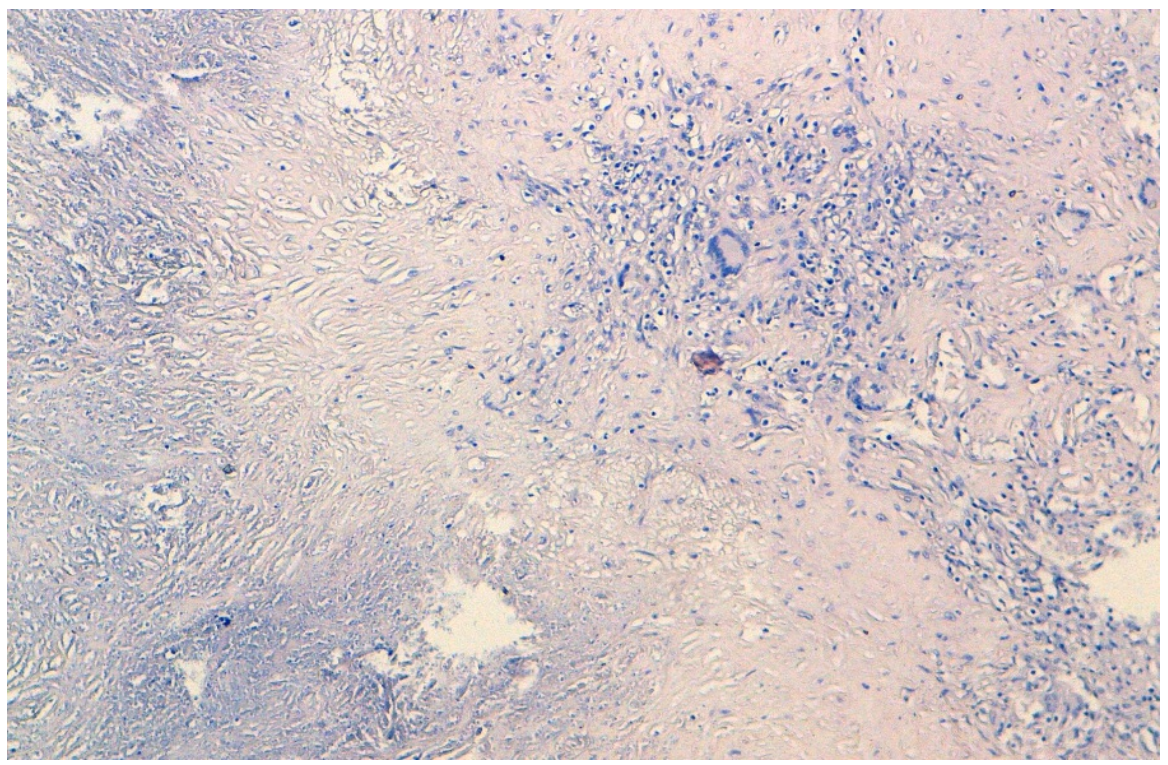

Figure 3. Granulomatous lesion with epithelioid cells and giant Langhans cells with caseating necrosis.

with good clinical improvement. At discharge there were no sign of meningismus or fever. The patient was symptom free at the last follow up exam at 14 months following the surgery.

\section{Discussion}

Intracranial tuberculomas are still common in developing countries [5]. They usually result from hematogenous spread of the mycobacterium tuberculosis to the leptomeninges and brain parenchyma from pulmonary tuberculosis [5]. En plaque Tuberculoma is a rare manifestation, with dural insertion, that appears as a solid mass that morphologically resembles a meningioma plaque or meningeal metastases [6] [7]. It was described for the first time in 1927 by Pardee and Knox as a meningeal process in plaque without exudation [1] [2]. It preferentially sits along the parietal and frontal convexity, tentorial, interhemispheric fissures and in the posterior fossa [8]. Macroscopically, it is a solid, encapsulated lesion diffusely infiltrating the dura with or without calcification [2].

Intracranial tuberculomas, have no clinical specificity, however one can find a syndrome of intracranial hypertension, seizures, focal weakness, neck stiffness with general symptoms like fever and anorexia [2] [6] [9] [10]. The extra neurological signs of tuberculosis are found in 30\% - 50\% [11].

They are usually seen in young adults and the pediatric population where they frequently occur in the infratentorial components as opposed to our case [6]. A pulmonary radiological abnormality is noted in $33 \%-50 \%$ of cases [2]. Tuberculin intradermal reaction is positive in $25 \%$ to $75 \%$ of patients [3]. CSF study most often shows a simple increase in protein [2] [10]. However, culture is not always conclusive [10].

On CT scan, they appear as dural based variably enhancing lesion with or 
without calcification, and often associated with active communicative hydrocephalus [12]. MRI is the investigation of choice and usually shows a hypointense lesion on T1 weighted, and a hypointense lesion with surrounding hyperintense edema on T2-weighted and enhances well with gadolinium contrast [4] [13]. In our case the lesion was multifocally infiltrating the brain parenchyma, and the enhancement pattern was heterogenous, but the dural insertion, the calcifications, the edema and the hydrocephalus were present. These finding along with the fever, neck stiffness and the high incidence of tuberculomas in our country allowed us to suspect a tuberculoma pre-operatively.

In most reported cases, the management included a surgical excision followed by antituberculous therapy and concomitant steroids administration as was the case in our patient [6] [9] [10] [14] [15] [16]. The frequently aggressive surgical resection approach toward en-plaque tuberculomas is probably due to the imprecise pre-operative diagnosis. Some authors emphasized the importance of a high index of suspicion due to the fact that medical therapy is generally effective for intracranial tuberculomas [17]. Previous reports described CT imaging of tuberculoma en plaque to be different from a meningioma, specifically the poorly defined density and the moderate contrast enhancement pattern in some cases [18] [19]. Singh et al. described the use of polymerase chain reaction assay and DNA amplification to diagnose en-plaque tuberculomas [20] with the objective to allow a trial with medical treatment with ATT and steroids. But in our experience and in the literature, a definite pre-operative diagnosis of tuberculoma en plaque remains challenging, specifically in the absence of extracranial tuberculosis [17]. Even in cases of high suspicion of intracranial tuberculoma like our patient, the mass effect, the concomitant active hydrocephalus, and the need for histologic confirmation make the surgical excision followed by medical therapy, a more reasonable approach. Most reported cases were confirmed post-operatively (including ours) by histology of the excised mass demonstrating granulomatous lesion with caseating necrosis and epithelioid and giant cells consistent with tuberculosis [6] [9] [10] [14] [15] [16]. There was a low complication rate following surgery and most reported cases experienced a full recovery [17].

\section{Conclusion}

In areas in which tuberculosis is endemic, en-plaque tuberculomas should be considered among the differential diagnosis of en-plaque mass lesions, especially when meningismus and clinical and radiological signs of hydrocephalus are present. The patients should be operated for surgical excision, ventriculoperitoneal shunting, and histologic confirmation of the diagnosis to avoid fatal outcomes.

\section{Patient Consent}

An informed written consent was obtained from the patient. 


\section{Conflicts of Interest}

The authors declare no conflicts of interest regarding the publication of this paper.

\section{References}

[1] Pardee, I. and Knox, L.C. (1927) Tuberculoma en plaque. Archives of Neurology \& Psychiatry, 17, 231-238. https://doi.org/10.1001/archneurpsyc.1927.02200320083006

[2] Elisevich, K. and Aprin, E.J. (1992) Tuberculoma Masquerading as a Meningioma. Journal of Neurosurgery, 56, 435-438. https://doi.org/10.3171/jns.1982.56.3.0435

[3] Chang, C.M., Chan, F.L., Yu, Y.L., Huang, C.Y. and Woo, E. (1986) Tuberculous Meningitis Associated with Meningeal Tuberculoma. Journal of the Royal Society of Medicine, 79, 486-487. https://doi.org/10.1177/014107688607900820

[4] Khanna, P.C., Godinho, S., Patkar, D.P., Pungavkar, S.A. and Lawande, M.A. (2006) MR Spectroscopy-Aided Differentiation: Giant Extra-Axial Tuberculoma Masquerading as Meningioma. American Journal of Neuroradiology, 27, 1438-1440.

[5] Ramachandran, R., Muniyandi, M., Iyer, V., Sripriya, T., Priya, B. and Govindarajan, T.G. (2017) Dilemmas in the Diagnosis and Treatment of Intracranial Tuberculomas. Journal of the Neurological Sciences, 381, 256-264. https://doi.org/10.1016/j.jns.2017.08.3258

[6] Dubey, S., Devi, B.I., Jawalkar, V.K. and Bhat, D.I. (2002) Tuberculoma en Plaque: A Case Report. Neurology India, 50, 497-499.

[7] Ng, F.C., Adamides, A.A. and Fabinyi, G.C. (2009) Atypical Meningeal Lesion. Journal of Clinical Neuroscience, 16, 1254-1254. https://doi.org/10.1016/j.jocn.2008.10.033

[8] Alkan, A., Parlak, M., Baysal, T., Sigirci, A., Kutlu, R. and Altinok, T. (2003) En-Plaque Tuberculomas of Tentorium in a Pregnant Woman: Follow-Up with MRI (2003:2b). European Radiology, 13, 1190-1193.

[9] Konstantin Elisevich, E. and Arpin, J. (1982) Tuberculoma Masquerading as a Meningioma: Case Report. Journal of Neurosurgery, 56, 435-438. https://doi.org/10.3171/jns.1982.56.3.0435

[10] Bauer, J., Johnson, R.F., Levy, J.M., Pojman, D.V. and Ruge, J.R. (1996) Tuberculoma Presenting as an en Plaque Meningioma. Journal of Neurosurgery, 85, 685-688. https://doi.org/10.3171/jns.1996.85.4.0685

[11] Dastur, H.M. and Desai, A.D. (1965) A Comparative Study of Brain Tuberculomas and Gliomas Based upon 107 Case Records of Each. Brain, 88, 375-396. https://doi.org/10.1093/brain/88.2.375

[12] Vengsarkar, U.S., Pisipaty, R.P., Parekh, B., et al. (1986) Intracranial Tuberculoma and the CT Scan. Journal of Neurosurgery, 64, 568-574. https://doi.org/10.3171/jns.1986.64.4.0568

[13] Indra, I.K. and Ganesan, S. (2001) Magnetic Resonance Imaging in Intracranial Tuberculosis. Medical Journal Armed Forces India, 57, 292-297. https://doi.org/10.1016/S0377-1237(01)80006-X

[14] Adachi, K., Yoshida, K., Tomita, H., Niimi, M. and Kawase, T. (2004) Tuberculoma Mimicking Falx Meningioma: Case Report. Neurologia Medico-Chirurgica, 44, 489-492. https://doi.org/10.2176/nmc.44.489

[15] Mirzai, H. (2005) Tuberculoma of the Cervical Spinal Canal Mimicking En Plaque 
Meningioma. Journal of Spinal Disorders \& Techniques, 18, 197-199. https://doi.org/10.1097/01.bsd.0000164733.32529.73

[16] Nayil, K., Kumar, K., Nair, S., Rao, R. and Menon, G. (2010) Dural Tuberculoma Mimicking En-Plaque Meningioma. Neurosurgery Quarterly, 20, 180-182. https://doi.org/10.1097/WNQ.0b013e3181eb2c29

[17] Kumar, J., Mallik, J., Strickland, B.A., Harsh, V. and Kumar, A. (2017) Intracranial En-Plaque Tuberculoma Impersonating En-Plaque Meningioma: Case Report and Review of Literature. Asian Journal of Neurosurgery, 12, 576-579. https://doi.org/10.4103/1793-5482.175619

[18] Welchman, J.M. (1979) Computerised Tomography of Intracranial Tuberculomata. Clinical Radiology, 30, 567-573. https://doi.org/10.1016/S0009-9260(79)80199-3

[19] Ng, S.H., Tang, L.M., Lui, T.N., Ko, S.F., Wong, H.F., Wai, Y.Y., et al. (1996) Tuberculoma en Plaque: CT. Neuroradiology, 38, 453-455. https://doi.org/10.1007/BF00607273

[20] Singh, K.K., Nair, M.D., Radhakrishnan, K. and Tyagi, J.S. (1999) Utility of PCR Assay in Diagnosis of En-Plaque Tuberculoma of the Brain. Journal of Clinical Microbiology, 37, 467-470. 